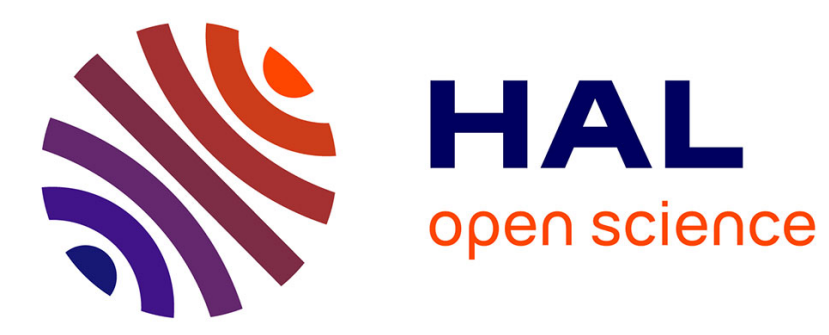

\title{
Particle Filtering for Online Space-Varying Blur Identification
}

\author{
Yunshi Huang, Emilie Chouzenoux, Víctor Elvira
}

\section{To cite this version:}

Yunshi Huang, Emilie Chouzenoux, Víctor Elvira. Particle Filtering for Online Space-Varying Blur Identification. CAMSAP 2019 - IEEE International Workshop on Computational Advances in MultiSensor Adaptive Processing, Dec 2019, Le Gosier, France. hal-02406970

\section{HAL Id: hal-02406970 https://hal.science/hal-02406970}

Submitted on 12 Dec 2019

HAL is a multi-disciplinary open access archive for the deposit and dissemination of scientific research documents, whether they are published or not. The documents may come from teaching and research institutions in France or abroad, or from public or private research centers.
L'archive ouverte pluridisciplinaire HAL, est destinée au dépôt et à la diffusion de documents scientifiques de niveau recherche, publiés ou non, émanant des établissements d'enseignement et de recherche français ou étrangers, des laboratoires publics ou privés. 


\title{
PARTICLE FILTERING FOR ONLINE SPACE-VARYING BLUR IDENTIFICATION
}

\author{
Yunshi Huang ${ }^{\dagger}$, Émilie Chouzenoux ${ }^{\dagger}$, and Víctor Elvira* \\ $\dagger$ CVN, INRIA Saclay, CentraleSupélec (France). \\ * IMT Lille Douai \& CRIStAL (UMR CNRS 9189), Villeneuve d'Ascq (France).
}

\begin{abstract}
The identification of parameters of spatially variant blurs given a clean image and its blurry noisy version is a challenging inverse problem of interest in many application fields, such as biological microscopy and astronomical imaging. In this paper, we consider a parametric form for the blur and introduce a state-space model that describes the statistical dependence among the neighboring kernels. Our Bayesian approach aims at estimating the posterior distributions of the kernel parameters given the available data. Since those posteriors are not tractable due to the nonlinearities of the model, we propose a sequential Monte Carlo approach to approximate the distributions by processing the data in an online manner. This allows to consider numerous overlapped patches and large scale images at reasonable computational and memory costs. Moreover, it provides a measure of uncertainty due to the Bayesian framework. Practical experimental results illustrate the good performance of our novel approach, emphasizing the benefit to exploit the spatial structure for an improved estimation quality.
\end{abstract}

Index Terms - Blur identification; spatially variant blur; Bayesian estimation; particle filtering.

\section{INTRODUCTION}

Optical instruments often produce images that suffer from blur due to light diffraction or object motion, among many other causes. The presence of the blur provokes an infinitesimally point-source to be spread in the acquired image defining the so-called Point Spread Function (PSF). Several computational strategies have been proposed in the literature for first estimating and then removing the blur in acquired images [1, 2], or for jointly estimating both image and blur with a blind deconvolution strategy [3]. Obviously, the performance of the deblurring step strongly depends on the accuracy of the PSF estimation. An efficient strategy for this estimation relies on a preliminary acquisition step of normalized and calibrated objects, such as fluorescent spherical microbeads in

The authors acknowledge support from the Agence Nationale de la Recherche of France under PISCES (ANR-17-CE40-0031-01) and MAJIC (ANR-17-CE40-0004-01) projects. microscopy [4] or resolution charts in digital camera calibration [5, 6]. Then, the problem is formulated as a linear system that can be inverted efficiently with penalized optimization strategies [7, 8]. The result can finally be fitted in a parametric non-linear model of the PSF in order to uncover characteristics of the optical system [9, 10, 11].

In most realistic scenarios, the PSF cannot be considered constant throughout the field-of-view due to various reasons such as defocus [12, 13], moving objects or cameras [14], anisotropic optical lens aberrations [15], or atmospheric turbulence [16, 17]. This non-stationary behavior gives rise to the so-called spatially variant blur [18]. This blur is much harder to estimate since the associated PSFs need to be recovered at each location in the spatial plane. This also induces a critical increase of computational complexity for identifying and removing the blur [7, 19, 20], and approximation strategies must often be employed to limit the cost of the blur operator [21, 22, 23].

In this paper, we focus on the problem of identifying the parameters of spatially variant PSFs from calibrated image acquisitions. We consider a parametric form for the blurs and formulate the problem of estimating the parameters as a state-space model assuming a smooth variation of the PSF shapes from neighboring regions. Since the resulting statespace model is nonlinear, we choose a particle filtering approach and its backward-simulation smoother to approximate the filtering and the smoothing posterior distributions, respectively. Our approach processes in an online manner a sequence of possibly overlapped patches extracted from the acquired image, and provides a probabilistic estimate of the kernel parameters at the corresponding patch locations. The Bayesian framework brings three key features, namely (i) limited computational cost and memory load thanks to an online processing, (ii) an explicit quantification of the statistical uncertainty on the estimated parameters, and (iii) a remarkable flexibility in the choice of the state-space models, allowing to account for non-linear relations and non-Gaussian noise. As an example, we propose a specific state-space model that accounts for smooth variations in scale and orientation along the neighboring kernels. We illustrate the efficiency and good performance of the approach by means of two sets of numerical experiments.

The rest of the paper is organized as follows. Section 2 
introduces the problem of spatially variant blur identification. Section 3 presents our algorithm for Bayesian inference, and introduces a specific state-space model for parametric PSF estimation. Section 4 illustrates the performance of the proposed approach, and Section 5 concludes the paper.

\section{PROBLEM STATEMENT}

Let us consider the observation of two images $(\mathbf{x}, \mathbf{y}) \in \mathbb{R}^{N}$, where $\mathbf{x}$ is the clean image and $\mathbf{y}$ is a blurry noisy version of it. Blur identification aims at estimating the parameters of the blur model that allows to describe the relationship between $\mathbf{x}$ and $\mathbf{y}$, accounting for the presence of noise. In this paper, we consider the case of spatially variant blurs. More precisely, let us decompose each image $\mathbf{x} \in \mathbb{R}^{N}$ into $T$ possibly overlapped patches $\left(\mathbf{x}_{t}\right)_{1 \leq t \leq T} \in \mathbb{R}^{P}$. We assume that, for each patch index $t \in\{1, \ldots, T\}$,

$$
\mathbf{y}_{t}=\mathbf{X}_{t} \mathbf{h}_{t}+\mathbf{n}_{t}
$$

where $\left(\mathbf{y}_{t}\right)_{1 \leq t \leq T} \in \mathbb{R}^{P}$ is the set of blurry noisy patches, $\left(\mathbf{n}_{t}\right)_{1 \leq t \leq T} \in \mathbb{R}^{P}$ models the additive noise. Hereabove, $\left(\mathbf{X}_{t}\right)_{1 \leq t \leq T} \in \mathbb{R}^{P \times L}$ are suitable block circulant matrices related to $\mathbf{x}$, which encode $2 \mathrm{D}$ convolutions with circulant boundaries padding with $\left(\mathbf{h}_{t}\right)_{1 \leq t \leq T} \in \mathbb{R}^{L}$, the unknown blur kernels associated with each patch localization. The goal is thus to estimate the set of kernels $\left(\mathbf{h}_{t}\right)_{1 \leq t \leq T}$, given the inputs $\left(\mathbf{y}_{t}, \mathbf{X}_{t}\right)_{1 \leq t \leq T}$. In this work, we will consider a parametric model for the blur kernels given by

$$
(\forall t \in\{1, \ldots, T\}), \quad \mathbf{h}_{t}=h\left(\boldsymbol{\rho}_{t}\right),
$$

where $h$ is a known function describing the general 2D shape of the kernels, parametrized by $\left(\boldsymbol{\rho}_{t}\right)_{1 \leq t \leq T} \in \mathbb{R}^{K}, K \geq 1$. For instance, a defocus blur can be described using a circular function parametrized by its radius [24], while uniform motion blur can depend on length and rotation parameters [25]. This parametric model can also encompass the widely used Gaussian blur shape [26] that we will retain for our experiments. More sophisticated parametric models can be found for instance in [27]. In the following section, we will propose a versatile filtering strategy for approximating the posterior distribution of $\left(\boldsymbol{\rho}_{t}\right)_{1 \leq t \leq T}$, while allowing for an efficient online treatment of the inputs $\left(\mathbf{y}_{t}, \mathbf{X}_{t}\right)_{1 \leq t \leq T}$. Then, we will discuss a useful example of state-space model to describe the statistical dependencies of the vector of parameters $\left(\boldsymbol{\rho}_{t}\right)_{1 \leq t \leq T}$.

\section{PROPOSED METHOD}

\subsection{Bayesian inference in state-space models}

Let us assume a Markovian dependency among the kernels, described by the state-space model

$$
p\left(\boldsymbol{\rho}_{0}\right), \quad p\left(\boldsymbol{\rho}_{t} \mid \boldsymbol{\rho}_{t-1}\right), \quad p\left(\mathbf{y}_{t} \mid \boldsymbol{\rho}_{t}, \mathbf{X}_{t}\right),
$$

Table 1: BPF algorithm with backward-simulation smoother for spacevariant blur identification.

1. Initialization. Draw $M$ i.i.d. samples, $\left(\boldsymbol{\rho}_{0}^{(m)}\right)_{1 \leq m \leq M}$ from the prior $p\left(\boldsymbol{\rho}_{0}\right)$.

2. Filtering step. For $t=1, \ldots, T$ :

(a) Simulate $\overline{\boldsymbol{\rho}}_{t}^{(m)} \sim p\left(\boldsymbol{\rho}_{t} \mid \boldsymbol{\rho}_{t-1}^{(m)}\right), \quad m=1, \ldots, M$.

(b) Compute the normalized weights by

$$
\bar{w}_{t}^{(m)} \propto p\left(\mathbf{y}_{t} \mid \overline{\boldsymbol{\rho}}_{t}^{(m)}, \mathbf{X}_{t}\right), \quad m=1, \ldots, M .
$$

(c) Append the samples to the state histories

$$
\hat{\boldsymbol{\rho}}_{0: t}^{(m)}=\left(\boldsymbol{\rho}_{0: t-1}^{(m)}, \overline{\boldsymbol{\rho}}_{t}^{(m)}\right), \quad m=1, \ldots, M .
$$

(d) Resample $M$ times from $\left\{\overline{\boldsymbol{\rho}}_{t}^{(m)}\right\}_{m=1}^{M}$ with associated probabilities $\left\{\bar{w}_{t}^{(m)}\right\}_{m=1}^{M}$, i.e., for $m=1, \ldots, M$, let $\boldsymbol{\rho}_{0: t}^{(m)}=\hat{\boldsymbol{\rho}}_{0: t}^{(j)}$ with probability $\bar{w}_{t}^{(j)}, j=1, \ldots, M$.

3. Backward-simulation smoothing step. For $s=1, \ldots, S$, choose

$$
\tilde{\boldsymbol{\rho}}_{T}^{(s)}=\overline{\boldsymbol{\rho}}_{T}^{(m)}
$$

with probability $\bar{w}_{T}^{(m)}$. Then for $t=T-1, \ldots, 1$ :

(a) Compute the normalized new weights by

$$
\bar{w}_{t \mid t+1}^{(m)} \propto \bar{w}_{t}^{(m)} p\left(\widetilde{\boldsymbol{\rho}}_{t+1} \mid \overline{\boldsymbol{\rho}}_{t}^{(m)}\right)
$$

(b) Choose

$$
\begin{aligned}
& \qquad \widetilde{\boldsymbol{\rho}}_{t}^{(s)}=\overline{\boldsymbol{\rho}}_{t}^{(m)} \\
& \text { with probability } \bar{w}_{t \mid t+1}^{(m)} .
\end{aligned}
$$

where $p\left(\boldsymbol{\rho}_{0}\right)$ is the prior distribution of the state, $p\left(\boldsymbol{\rho}_{t} \mid \boldsymbol{\rho}_{t-1}\right)$ is the state model, and $p\left(\mathbf{y}_{t} \mid \boldsymbol{\rho}_{t}, \mathbf{X}_{t}\right)$ is the observation model. Our goal consists in estimating probabilistically the unknown parameters of the model. In the sequential processing approach, we build the sequence of filtering posterior distributions given the processed data up to patch index $t$, $\left(p\left(\boldsymbol{\rho}_{t} \mid \mathbf{X}_{1: t}, \mathbf{y}_{1: t}\right)\right)_{1 \leq t \leq T}$ and the sequence of smoothing posterior distributions given all dataset $\left(p\left(\boldsymbol{\rho}_{t} \mid \mathbf{X}_{1: T}, \mathbf{y}_{1: T}\right)\right)_{1 \leq t \leq T}$. Unfortunately, for most realistic state-space models, these distributions cannot be obtained analytically, and one needs to resort to approximations. Table 1 describes the sequential Monte Carlo approach for estimating those distributions. Step 2 implements the filtering mechanism, and in particular we describe the bootstrap particle filter (BPF) algorithm. In Step 3, we implement the backward-simulation (BS) smoother to approximate the smoothing distribution, given all filtering distributions. Note that, without loss of generality, we have considered the BPF (arguably the most popular particle filter implementation [28]) and the simple BS smoother, but other advanced filters could be employed instead [29, 30, 31]. As a result of the BPF, for each patch $t$, we can approximate the filtering distribution $p\left(\boldsymbol{\rho}_{t} \mid \mathbf{X}_{1: t}, \mathbf{y}_{1: t}\right)$ by the $M$ generated particles $\left(\overline{\boldsymbol{\rho}}_{t}^{(m)}\right)_{1 \leq m \leq M}$ and associated normalized weights $\left(\bar{w}_{t}^{(m)}\right)_{1 \leq m \leq M}$ computed in Step 2(a)-(b). Moreover, the BS step allows to approximate the smoothing posterior distributions $\left(p\left(\boldsymbol{\rho}_{t} \mid \mathbf{X}_{1: T}, \mathbf{y}_{1: T}\right)\right)_{1 \leq t \leq T}$ by $S$ equally weighted 
sampled particles $\left(\widetilde{\boldsymbol{\rho}}_{t}^{(s)}\right)_{1 \leq s \leq S}$ given by Step 3(b).

\subsection{State-space model for the PSF variation}

Let us now present a specific model for the evolution of the parameters $\left(\boldsymbol{\rho}_{t}\right)_{1 \leq t \leq T}$ along the patches, which relies on the assumption that the PSFs of two neighboring patches only differ by a small change in their scale and orientation. More precisely, let us consider that function $h(\cdot)$ takes as an input a vector of three parameters $(\theta, \mathbf{s})$. The parameter $\theta \in \mathbb{R}$ defines the orientation while $\mathbf{s} \in(0,+\infty)^{2}$ quantifies the width of the PSF. This model allows for instance to encompass the family of centered Gaussian blur kernels parametrized by a covariance matrix $\mathbf{C}(\theta, \mathbf{s})=\mathbf{R}_{\theta} \operatorname{Diag}(\mathbf{s}) \mathbf{R}_{\theta}^{\top}$ with $\mathbf{R}_{\theta} \in \mathbb{R}^{2 \times 2}$ denoting the rotation matrix with angle $\theta$. We then propose the following Markov model for $\left(\boldsymbol{\rho}_{t}\right)_{1 \leq t \leq T}=\left(\left[\theta_{t}, \mathbf{s}_{t}\right]\right)_{1 \leq t \leq T}$ :

$$
p\left(\theta_{t} \mid \theta_{t-1}\right)=\mathcal{N}\left(\theta_{t} ; \theta_{t-1}, \sigma_{\theta}^{2}\right),
$$

with $\sigma_{\theta}>0$, and

$$
p\left(\mathbf{s}_{t} \mid \mathbf{s}_{t-1}\right)=\mathcal{N}_{\left[s_{\text {min }}, s_{\max }\right]}\left(\mathbf{s}_{t} ; \mathbf{s}_{t-1}, \sigma_{s}^{2} \mathbf{I}\right),
$$

where $\sigma_{s}>0$ and $\left[s_{\min }, s_{\max }\right] \subset(0,+\infty)$ is the support of the truncated Gaussian distribution that models the scale parameters evolution. Parameters $\sigma_{\theta}, \sigma_{s}, s_{\min }$, and $s_{\max }$ are assumed to be known. Note that there exist methods that can estimate these static parameters otherwise [32].

\section{EXPERIMENTAL RESULTS}

\subsection{Experimental settings}

We illustrate the performance of the proposed approach in two sets of numerical experiments. Three images of size $N=$ $512 \times 512$ displayed in Fig. 1 will be considered in our tests (Mire, Cells, and Hubble), which represent objects that are typically imaged for blur calibration in digital cameras, microscopes, and telescopes, respectively. Each image $\mathbf{x}$ is decomposed into $T$ possibly overlapped patches of size $64 \times$ 64 . Each patch $\left(\mathbf{y}_{t}\right)_{1 \leq t \leq T}$ of the blurry noisy image $\mathbf{y}$ is generated using Eqs. (1)-(2). For each patch, $h\left(\boldsymbol{\rho}_{t}\right)$ is a normalized Gaussian blur kernel of size $15 \times 15$ with mean at the center $(8,8)$, and with both width and orientation parametrized by the covariance matrix $\mathbf{C}\left(\theta_{t}, \mathbf{s}_{t}\right)$ introduced above. Parameters $\left(\theta_{t}, \mathbf{s}_{t}\right)_{1 \leq t \leq T}$ to be estimated are chosen using two alternative strategies described in the next subsection. The prior distribution in the state-space model is considered as uniform: $p\left(\theta_{0}\right)=\mathcal{U}([0,2 \pi]), p\left(\mathbf{s}_{0}\right)=\mathcal{U}\left(\left[s_{\min }, s_{\max }\right]^{2}\right)$. For simplicity we consider a Gaussian noise model given by $p\left(\mathbf{y}_{t} \mid \boldsymbol{\rho}_{t}, \mathbf{X}_{t}\right)=\mathcal{N}\left(\mathbf{y}_{t} ; \mathbf{X}_{t} h\left(\boldsymbol{\rho}_{t}\right), \sigma_{n}^{2} \mathbf{I}\right)$. More sophisticated noise models could be directly plugged into our framework (see for instance [33]), since the only requirement of the BPF is to be able to evaluate the likelihood functional in Step 2(b)
[30]. We implement BPF algorithm with $M=1000$ particles and $S=100$ samples for BS as this setting allows to reach the best compromise in terms of computational complexity and stability of the estimator performance. BPF algorithm followed by a BS step is noted as BPF-BS. More advanced particle filters could be directly used, e.g., auxiliary PFs [29, 34] or schemes with an automatic adaptation of $M$ [35]. Finally, note that the numbering order for the patches plays an important role in our method, as it dictates the ordering relation in our Markovian transition model, as well as the order of the sequential updates in Table 1 If not specified otherwise, we will follow the lexicographic ordering. The estimation of spatially variant PSF parameters in an online manner has not been addressed in the literature, up to our knowledge. In order to quantify the gain of exploiting the spatial structure of the problem, we compare the filtering and smoothing posteriors with the posterior distribution $p\left(\boldsymbol{\rho}_{t} \mid \mathbf{X}_{t}, \mathbf{y}_{t}\right)$, approximated by an importance sampling (IS) strategy. We also compare with an approach based on the maximum a posteriori (MAP) estimator of $\left(\mathbf{h}_{t}\right)_{1 \leq t \leq T}$ that promotes space-varying blurs with smooth variations, satisfying simplex constraint [36, 7]. Note that no parametric model is assumed in that case. More precisely, a penalized least squares loss function, accounting for simplex constraints as well as 2D spatial regularity, is formulated, and minimized using FISTA algorithm [37].

The performance of all methods are evaluated in terms of the root mean squared error (RMSE) averaged over patches, i.e., $\mathrm{RMSE}=\frac{1}{T} \sum_{t=1}^{T} \frac{\left\|\overline{\mathbf{h}}_{t}-\mathbf{h}_{t}\right\|_{2}}{\left\|\mathbf{h}_{t}\right\|_{2}}$ where $\mathbf{h}_{t}$ and $\overline{\mathbf{h}}_{t}$ are respectively the original and estimated ke also promotes a spatiallyvarying blurrnels at patch $t$. More precisely, $\overline{\mathbf{h}}_{t}=h\left(\bar{\theta}_{t}, \overline{\mathbf{s}}_{t}\right)$ with $\bar{\theta}_{t}$ and $\overline{\mathbf{s}}_{t}$ the mean estimators of the angle and scale parameters for BPF, BPF-BS and IS, while $\overline{\mathbf{h}}_{t}$ is the MAP estimator at patch $t$, for the MAP approach. Note that BPF, BPF$\mathrm{BS}$, and IS approximate the whole distribution of the patch parameters, so any other moment of the distribution could be computed, contrasting with the MAP strategy.

All the presented results are averaged over 100 runs. We conduct the numerical experiments in a Matlab environment on a computer with an Xeon $(\mathrm{R}) \mathrm{W}-2135$ processor $(3.7 \mathrm{GHz}$ clock frequency) and $12 \mathrm{~GB}$ of RAM.

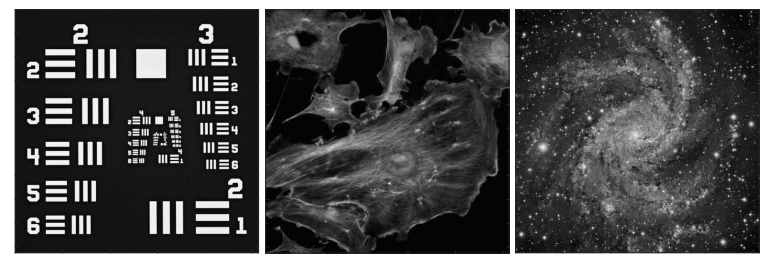

Fig. 1: Test images Mire, Cells and Hubble.

\subsection{Numerical results}

In our first set of experiments, parameters $\left(\theta_{t}, \mathbf{s}_{t}\right)_{1 \leq t \leq T}$ are generated following the transition model in Section 3.2 . with $T=64$ non-overlapped patches, $\sigma_{\theta}=\sigma_{s}=0.1$, $s_{\min }=0.01$ and $s_{\max }=0.5$. Two noise levels are consid- 
ered, namely $\sigma_{n} \in\{0.01,0.1\}$. Table 2 presents the averaged RMSE for all tested methods. A first observation is that BPF, BPF-BS and IS appear superior to the MAP approach in terms of accuracy, probably because they account explicitly for the parametric shape of the blur. Note that the former methods are also faster. In this example, the running time for MAP is 160 seconds, 10 seconds for BPF and IS and 22 seconds for BPF-BS. The poor performance of IS illustrates that adding the information of the spatial structure improves the inference task. Finally, the smoothing procedure increases the quality of the estimates, at the expense of an extra pass on the dataset and hence a slight increase of computational time. Figure 2 displays, for a specific run, the true parameters (blue circles), the mean of the posterior approximated by BPF (red crosses) and BPF-BS (green crosses), and the error bars showing the mean plus/minus two standard deviations approximated by BPF (red lines) and BPF-BS (green lines). The figure not only shows the great tracking ability of both filtering/smoothing methods but also the uncertainty quantification (note that in patches where the estimation is not as good, the error bars are also wide). One can notice that BPF-BS has improved estimates for the first patch when compared to the BPF thanks to the backward-simulation smoothing process.

\begin{tabular}{|c|l||c|c|c|}
\hline$\sigma_{n}$ & & Mire & Cells & Hubble \\
\hline \hline \multirow{3}{*}{$10^{-2}$} & BPF & $\mathbf{0 . 0 8 2 5}$ & $\mathbf{0 . 0 7 3 3}$ & $\mathbf{0 . 0 6 8 2}$ \\
& BPF-BS & $\mathbf{0 . 0 8 2 7}$ & $\mathbf{0 . 0 7 5 4}$ & $\mathbf{0 . 0 6 6 3}$ \\
& IS & 0.1373 & 0.1892 & 0.1504 \\
& MAP & 0.1870 & 0.2462 & 0.1905 \\
\hline \multirow{3}{*}{$10^{-1}$} & BPF & 0.1409 & 0.2202 & 0.1304 \\
& BPF-BS & $\mathbf{0 . 1 2 6 9}$ & $\mathbf{0 . 1 4 3 4}$ & $\mathbf{0 . 1 1 2 7}$ \\
& IS & 0.2491 & 0.3493 & 0.2877 \\
& MAP & 0.2923 & 0.3451 & 0.3380 \\
\hline
\end{tabular}

Table 2: RMSE for BPF, BPF-BS, IS and MAP approaches respectively. Kernels generated using the exact transition models.
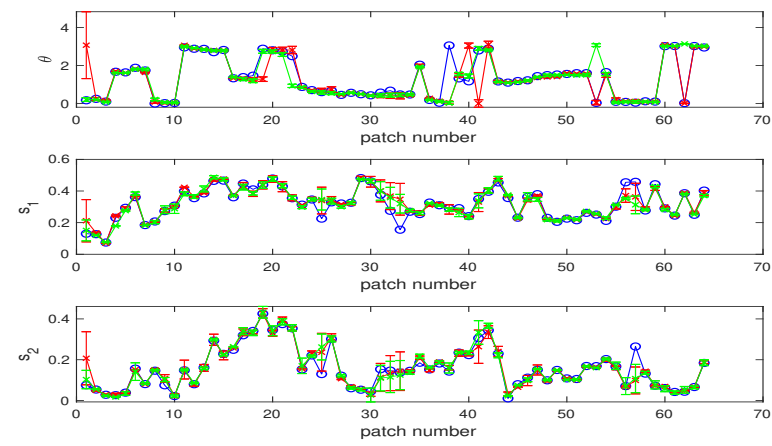

Fig. 2: Mean and variance estimates using BPF (red) and BPF-BS (green) compared to the original parameters generated using the state-space model (blue) for noise level 0.1 and Mire image.

In the second setup, the scale and orientation parameters of the kernels are generated using realistic optical models. Two spatially variant models described in [23, Fig.8] and [2, Fig.7] are tested. Small random perturbations are added in the parameters of the evolution laws explaining the spatial variation of the PSFs to better represent the variability one can experience in a true acquisition device. Regarding the patch
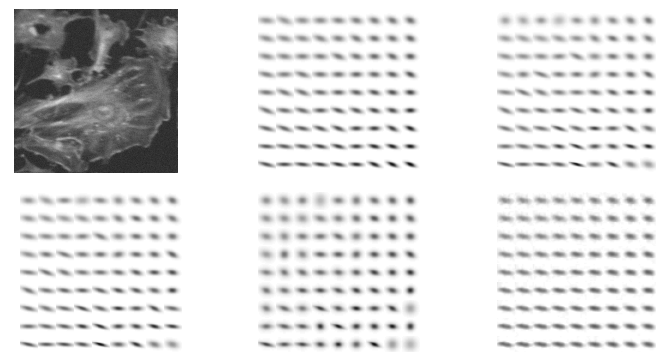

Fig. 3: Kernels identification using Cells image with $8 \times 8$ overlap: noisy blurry images (top-left); original kernels generated using model from [2] Fig.7] (top-middle); restored kernels with BPF (top-right), BPF-BS (bottomleft), IS (bottom-middle) and MAP (bottom-right).

\begin{tabular}{|c|l||c|c|c|}
\hline Overlapped size & & $0 \times 0$ & $8 \times 8$ & $32 \times 32$ \\
\hline \hline \multirow{2}{*}{ Model [23] Fig.8] } & BPF & 0.1663 & 0.1414 & 0.1151 \\
& BPF-BS & $\mathbf{0 . 1 2 5 8}$ & $\mathbf{0 . 1 1 0 7}$ & $\mathbf{0 . 1 0 2 3}$ \\
& IS & 0.2883 & 0.2829 & 0.2808 \\
& MAP & 0.2543 & 0.2380 & 0.2098 \\
\hline \multirow{2}{*}{ Model [2] Fig.7] } & BPF & 0.1313 & 0.1191 & 0.1028 \\
& BPF-BS & $\mathbf{0 . 1 0 7 9}$ & $\mathbf{0 . 1 0 5 4}$ & $\mathbf{0 . 0 9 3 2}$ \\
& IS & 0.3361 & 0.3321 & 0.3313 \\
& MAP & 0.1608 & 0.1641 & 0.1525 \\
\hline
\end{tabular}

Table 3: RMSE for BPF, BPF-BS, IS, MAP respectively. Cells image using kernels generated with two realistic space-varying models.

positions, we consider three cases, namely (i) non-overlapped ( $T=64)$, (ii) overlapped size $=8 \times 8(T=81)$ and (iii) overlapped size $=32 \times 32(T=225)$. The spiral order initialized in the image center is used for the model from [23, Fig.8] to better capture the radial symmetry of the kernels map. We set $\sigma_{n}=0.05$ and $\left(\sigma_{\theta}, \sigma_{s}\right)$ are chosen using a golden search to minimize the RMSE. Table 3 presents RMSE for BPF, BPF-BS, IS and MAP approaches for image Cells. Again, we clearly observe the improvement brought by our strategy. Moreover, as expected, increasing the overlapped size tends to improve the results quality, as more observations facilitate the inference task. Fig. 3 presents an example of results that illustrates the spatial regularization effect brought by the proposed state model, which contrasts with the non-regularized IS methods. We can also visually assess the advantage of using a parametric form for the kernel, when compared to the non-parametric MAP approach. The estimators derived by BPF-BS are the best among these four methods in all cases.

\section{CONCLUSION}

This paper addresses the estimation of PSF parameters for spatially varying blurs. We propose an original statistical modeling of the problem, accounting for the spatial dependency among neighboring kernels, and apply a sequential Bayesian inference technique in this context. Our results in different scenarios illustrate the good performance of the approach, including a useful uncertainty quantification. The novel approach opens many possibilities beyond this work. For instance, different noise distributions could be immediately used. Moreover, other state-space models, not necessarily Markovian, could be considered. 


\section{REFERENCES}

[1] N. Pustelnik, A. Benazza-Benhayia, Y. Zheng, and J.-C. Pesquet, "Wavelet-based image deconvolution and reconstruction," 2016, Wiley Encyclopedia of Electrical and Electronics Engineering.

[2] S. Berisha and J. G. Nagy, Iterative Methods for Image Restoration, chapter 4, pp. 193-247, 2014.

[3] A. Levin, Y. Weiss, F. Durand, and W. T. Freeman, "Understanding blind deconvolution algorithms," IEEE Transactions on Pattern Analysis and Machine Intelligence, vol. 33, no. 12, pp. 2354-2367, Dec. 2011.

[4] H. Kirshner, T. Pengo, N. Olivier, D. Sage, S. Manley, and M. Unser, "A psf-based approach to biplane calibration in 3d super-resolution microscopy," in 9th IEEE Int. Symp. Biomed. Imaging (ISBI 2012), Barcelona, Spain, 2-5 May 2012, pp. 1232-1235.

[5] F. Mannan and M. S. Langer, "Blur calibration for depth from defocus," in Proceedings of 13th Conference on Computer and Robot Vision (CRV 2016), June 2016, pp. 281-288.

[6] T. Bell, J. Xu, and S. Zhang, "Method for out-of-focus camera calibration," Applied Optics, vol. 55, no. 9, pp. 2346-2352, Mar 2016.

[7] R. Tezaur, T. Kamata, H.Li, and S.S. Slonaker, "A system for estimating optics blur psfs from test chart images," in Proceedings of SPIE - The International Society for Optical Engineering. IEEE, 2015, vol. 9404.

[8] E. Chouzenoux and J.-C. Pesquet, "A stochastic majorize-minimize subspace algorithm for online penalized least squares estimation," IEEE Transactions on Signal Processing, vol. 65, no. 18, pp. 47704783, 2017.

[9] S. M. Anthony and S. Granick, "Image analysis with rapid and accurate two-dimensional Gaussian fitting," Langmuir, vol. 25, no. 14, pp. 8152-8160, 2009.

[10] E. Chouzenoux, T.T.K. Lau, C. Lefort, and J.C. Pesquet, "Optimal multivariate gaussian fitting with applications to PSF modeling in twophoton microscopy imaging," Journal of Mathematical Imaging and Vision, 2019

[11] H. Kirshner, F. Ahuet, D. Sage, and M. Unser, "3-D PSF fitting for fluorescence microscopy: implementation and localization application," J. Microsc., vol. 249, no. 1, pp. 13-25, 2013.

[12] P. Trouvé, F. Champagnat, G. Le Besnerais, and J. Idier, "Single image local blur identification," in 18th IEEE International Conference on Image Processing (ICIP 2011). Brussels, 2011, pp. 613-616.

[13] X. Zhang, R. Wang, X. Jiang, W. Wang, and W. Gao, "Spatially variant defocus blur map estimation and deblurring from a single image," Journal of Visual Communication and Image Representation, vol. 35, 2016.

[14] H. Zhe, X. Li, and M.H. Yang, "Joint depth estimation and camera shake removal from single blurry image," in IEEE Conference on Computer Vision and Pattern Recognition (CVPR 2014). Cairo, 2014, pp. 2893-2900.

[15] A. Jezierska, H. Talbot, and J.C.Pesquet, "Spatially variant psf modeling in confocal macroscopy," in IEEE 15th International Symposium on Biomedical Imaging (ISBI 2018). Washington, DC, 2018, pp. 489-492.

[16] D. Li, R. M. Mersereau, and S. Simske, "Atmospheric turbulencedegraded image restoration using principal components analysis," IEEE Geoscience and Remote Sensing Letters, vol. 4, no. 3, pp. 340344, 2007.

[17] M A. Schmitz et al., "Euclid: Non-parametric point spread function field recovery through interpolation on a graph laplacian," 062019.

[18] A. Chakrabarti, T. Zickler, and W. T. Freeman, "Analyzing spatiallyvarying blur," in 2010 IEEE Computer Society Conference on Computer Vision and Pattern Recognition. San Francisco, CA, 2010, pp. 2512-2519.
[19] R. H. Chan, T. F. Chan, L. Shen, and Z. Shen, "Wavelet deblurring algorithms for spatially varying blur from high-resolution image reconstruction," Linear Algebra and its Applications, vol. 366, pp. 139 155, 2003, Special issue on Structured Matrices: Analysis, Algorithms and Applications.

[20] S. Ben Hadj, L. Blanc-Féraud, and G. Aubert, "Space variant blind image restoration," SIAM Journal on Imaging Sciences, vol. 7, 2014.

[21] F. Sroubek, J. Kamenicky, and Y. M. Lu, "Decomposition of spacevariant blur in image deconvolution," IEEE Signal Processing Letters, vol. 23, pp. 346-350, 2016.

[22] L. Denis, E. Thiébaut, F. Soulez, J.M. Becker, and R. Mourya, "Fast approximations of shift-variant blur," International Journal of Computer Vision, vol. 115, pp. 253-278, 2015.

[23] P. Escande and P. Weiss, "Sparse wavelet representations of spatially varying blurring operators," SIAM Journal on Imaging Sciences, vol. 8, pp. 2976-3014, 2015.

[24] X. Zhu, S. Cohen, S. Schiller, and P. Milanfar, "Estimating spatially varying defocus blur from a single image," IEEE Transactions on Image Processing, vol. 22, no. 12, pp. 4879-4891, 2013.

[25] R. Lokhande, K.V. Arya, and P. Gupta, "Identification of parameters and restoration of motion blurred images," in Proceedings of the 2006 ACM Symposium on Applied Computing (SAC 2006), Dijon, France, 23-27 April 2006, p. 301-315.

[26] B. Zhang, J. Zerubia, and J.-C. Olivo-Marin, "Gaussian approximations of fluorescence microscope point-spread function models," Appl. Opt., vol. 46, no. 10, pp. 1819-1829, Apr 2007.

[27] Robert L. Stevenson Jonathan D. Simpkins, "Parameterized modeling of spatially varying optical blur," Journal of Electronic Imaging, vol. 23, no. 1, pp. $1-13-13,2014$.

[28] N. Gordon, D. Salmond, and A. F. M. Smith, "Novel approach to nonlinear and non-Gaussian Bayesian state estimation," IEE ProceedingsF Radar and Signal Processing, vol. 140, pp. 107-113, 1993.

[29] M. K. Pitt and N. Shephard, "Auxiliary variable based particle filters," in Sequential Monte Carlo Methods in Practice, A. Doucet, N. de Freitas, and N. Gordon, Eds., chapter 13, pp. 273-293. Springer, 2001.

[30] P. M. Djurić, J. H. Kotecha, J. Zhang, Y. Huang, T. Ghirmai, M. F. Bugallo, and J. Míguez, "Particle filtering," IEEE Signal Processing Magazine, vol. 20, no. 5, pp. 19-38, September 2003.

[31] A. Doucet and A. M. Johansen, "A tutorial on particle filtering and smoothing: Fifteen years later," Handbook of nonlinear filtering, vol. 12, no. 656-704, pp. 3, 2009.

[32] C. Andrieu, A. Doucet, and R. Holenstein, "Particle Markov Chain Monte Carlo methods," Journal of the Royal Statistical Society: Series B (Statistical Methodology), vol. 72, no. 3, pp. 269-342, 2010.

[33] E. Chouzenoux Y. Marnissi, Y. Zheng and J.-C. Pesquet, "A variational bayesian approach for image restoration. application to image deblurring with Poisson-Gaussian noise," IEEE Transactions on Image Computing, vol. 3, no. 4, pp. 722-737, May 2017.

[34] V. Elvira, L. Martino, M. F Bugallo, and P. M. Djurić, "In search for improved auxiliary particle filters," in 2018 26th European Signal Processing Conference (EUSIPCO). IEEE, 2018, pp. 1637-1641.

[35] V. Elvira, J. Míguez, and P. M. Djurić, "Adapting the number of particles in sequential monte carlo methods through an online scheme for convergence assessment," IEEE Transactions on Signal Processing, vol. 65, no. 7, pp. 1781-1794, 2017.

[36] R. K. Mourya, "Contributions to image restoration : from numerical optimization strategies to blind deconvolution and shift-variant deblurring," PhD thesis, 2016, https://tel.archives-ouvertes. fr/tel-01764912

[37] A. Beck and M. Teboulle, "A fast iterative shrinkage-thresholding algorithm for linear inverse problems," SIAM J. Imaging Sciences, vol. 2, pp. 183-202, 012009. 